\title{
Effects of Graphenes/CNTs Co-reinforcement on Electrical and Mechanical Properties of HDPE Matrix Nanocomposites
}

\author{
Byung-Joo Kim, Joon-Hyung Byun, ${ }^{\dagger}$ and Soo-Jin Park ${ }^{\dagger, *}$ \\ Nano Material Research Dept., Jeonju Institute of Machinery and Carbon Composites, Palbokdong-2ga, 817, Jeonju, \\ Jeollabuk-do 561-844, Korea \\ †Dept. of Chemistry, Inha Univ., Incheon 402-751, Korea. "E-mail: sjpark@inha.ac.kr \\ ${ }^{\star}$ Composite Materials Lab, Korea Institute of Machinery \& Materials, 66 Sangnam-dong, Changwon 641-831, Korea \\ Received January 26, 2010, Accepted June 18, 2010
}

\begin{abstract}
In this work, mechanical and electrical properties of graphenes (GP)/carbon nanotubes (CNTs) co-reinforced high density polyethylene (HDPE) matrix composites were studied. The microstructure, morphologies, and electric properties of the composites were evaluated by XRD, TEM, and 4-probe methods, respectively. It was found that the electric resistivity of $0.5 \mathrm{wt} \%$-GP/HDPE was immeasurable, and $2.0 \mathrm{wt} \%$-CNTs/HDPE showed high resistivity $\left(6.02 \times 10^{4} \Omega \cdot \mathrm{cm}\right)$. Meanwhile, GP $(0.5 \mathrm{wt} \%) / \mathrm{CNTs}(2.0 \mathrm{wt} \%) / \mathrm{HDPE}$ showed excellent low resistivity $\left(3.1 \times 10^{2} \Omega \cdot \mathrm{cm}\right)$. This result indicates that the co-reinforcement systems can dramatically decrease electric resistivity of the carbon/polymer nanocomposites.
\end{abstract}

Key Words: Co-reinforcements, Graphenes, Carbon nanotubes, Nanocomposites

\section{Introduction}

Many metallic materials have been replaced by conductive polymers that have several advantages such as high specific strength and modulus, low coat and high density, renewable nature, and effective processing. ${ }^{1-5}$ Their utilizable advantages can be applied to microwave interception, functional cloths, electromagnetic field interference shielding (EMI), cathode materials for fuel cells, semi-conductive polymers, reinforced materials, and industrial applications of motor vehicles and aircrafts. ${ }^{1,2}$

Carbon materials with good electric conductivity (low resistivity), such as graphites, multi-walled carbon nanotubes, or single-walled carbon nanotubes, and carbon nanofibers, have been investigated as conductive fillers in polymer matrices in order to promote value added and large applications of conductive polymer composites. ${ }^{3,6-11}$

Normally, CNTs have very low electrical resistivity (or high conductivity) (specific electric resistivity: $\left.10^{-3} \sim 10^{-2} \Omega \mathrm{cm}\right)^{6}$ and it is possible to apply them as EMI shielding materials, which need to be in the range of $10^{2} \sim 10^{-1} \Omega \mathrm{cm}$ of specific resistance, corresponding to $30 \sim 40 \mathrm{~dB}$ of shielding performance. ${ }^{12-14}$ One of main obstacles for CNT applications in the conductive composites is difficulty of dispersion in polymer matrices. To solve this problem, many researchers have traditionally tried to disperse CNTs in the matrices by several surface treatment methods or in situ polymerization, but these remain as a key problem of CNT applications. ${ }^{6}$

It is well-known that graphites with high crystallinity have very low electric resistivity $\left(10^{-5} \Omega \mathrm{cm}\right)$. However, if graphite sheets (or graphenes) are used as single conductive fillers in polymer matrices, it is hard to get good electric conductivity due to the bad electric networks in the matrices. Lu et al. ${ }^{15}$ reported that graphites/HDPE could have a good electric conductivity by high pressure pressing due to the formation of new electric networks. Basically, graphites are composed of many sheets of graphene layer, so the graphite can be applied as layered filler material or graphenes by single-sheet-expanding methods. The acid treatment followed by steep thermal treatment method is well-known as a top-down method for graphene preparation. Normally, acid solutions were intercalated between graphite layers during acid treatment process. And severe heat treatment conditions (over $1000{ }^{\circ} \mathrm{C}$, exposing time $1 \sim 5 \mathrm{~min}$ ) lead to the steep vaporization of acid solutions in the graphite, resulting in the formation of full exfoliated graphites, so-called graphite nanoplatelet or graphenes (The graphite layer number classify each product.).

Graphenes have good potential as conductive fillers when they have a high electric conductivity and can make suitable electric networks in the matrices. Practically, it is very hard to achieve maintenance of successfully connected electric networks in polymer matrices.

Therefore, it is possible to think that if CNTs and GP are employed as conductive co-fillers in polymer matrices, CNTs can help to form good electric networks by connecting each graphene layers in the matrices.

In this respect, we prepared GP/CNTs/HDPE nanocomposites as conductive materials that can be used for the EMI shielding. The synergetic effects of GP and CNTs were evaluated by the electric conductivity and mechanical properties of the nanocomposites.

\section{Materials and Methods}

Materials and sample preparation. High density polyethylene (HDPE) and carbon nanotubes (CNTs) were purchased from Honam Petrochemical Co. (Mw: 500,000) and Nano Solution Co., Ltd., respectively. Graphite flakes were provided from Aldrich Co. Sulfuric acid and hydrogen peroxide were purchased from Jin Chemical Pharmaceutical Co., Ltd. Graphenes (GP) 
Table 1. HDPE with composition in a different weight ratio

\begin{tabular}{lcc}
\hline \multicolumn{1}{c}{ Sample name } & GP (wt \%) & CNTs (wt \%) \\
\hline Pure HDPE & - & - \\
CNTs-2.0/HDPE & - & 2.0 \\
GP-0.5/HDPE & 0.5 & - \\
GP-0.5/CNTs-2.0/HDPE & 0.5 & 2.0 \\
GP-0.5/CNTs-4.0/HDPE & 0.5 & 4.0 \\
\hline
\end{tabular}

were prepared by the following methods. $20 \mathrm{~g}$ of graphite flakes were dipped in the mixed acid solution $\left(\mathrm{H}_{2} \mathrm{SO}_{4}, 5: \mathrm{H}_{2} \mathrm{O}_{2}, 1\right)$ for $12 \mathrm{~h}$ at room temperature. After this, graphites were thermally treated in a furnace at $700{ }^{\circ} \mathrm{C}$ for $2 \mathrm{~min}\left(50 \mathrm{cc} / \mathrm{min}\right.$ of $\mathrm{N}_{2}$ gas $)$, and then the graphenes were washed with distilled water three times. To purify CNTs, they were oxidized in a furnace for 30 min at $300{ }^{\circ} \mathrm{C}$ with air stream, and then dipped in high concentration $\mathrm{HNO}_{3}$ solution for $30 \mathrm{~min}$.

For the composite production, HDPE was melt-blended at $150{ }^{\circ} \mathrm{C}$ for $1 \mathrm{~h}$ with the addition of various GP and CNT content in the matrix. Table 1 presents the mixing ratio of the GP/CNTs/ HDPE composites.

Measurements. Wide-angle X-ray diffraction (XRD) patterns of the nanocomposites were obtained with a Rigaku Model D/MAX-III B diffraction meter equipped with a rotation anode, using $\mathrm{Cu} \mathrm{K} \alpha$ radiation $(\lambda=0.15418 \mathrm{~nm})$.

TGAs were performed, using a du Pont TGA-2950 analyzer, to investigate the thermal stabilities of the composites from $25{ }^{\circ} \mathrm{C}$ to $600{ }^{\circ} \mathrm{C}$ at the heating rate of $10{ }^{\circ} \mathrm{C} / \mathrm{min}$ in a nitrogen atmosphere.

A JEM 2100F transmission electron microscope (TEM) was used to observe the morphologies of graphene layers and CNTs in the HDPE matrix.

The tensile strength of the composites was measured on a universal tester (Instron Model 1125 mechanical tester) according to ASTM D 638-95. All of the mechanical property values were obtained by averaging the five experimental values. The sample size was $2 \mathrm{~mm} \times 25 \mathrm{~mm} \times 75 \mathrm{~mm}$ and the crosshead speed was $1 \mathrm{~mm} / \mathrm{min}$.

The electric resistivity of the composites was determined by measuring the volume resistivity, which was measured at room temperature using a digital multimeter (MCP-T610, Mitsubishi Chemical Cooperation of Japan).

\section{Results and Discussion}

XRD studies. XRD analysis was employed to examine changes of crystal structures of the GP/CNTs/HDPE nanocomposites with different filler contents and composition. In Figure 1, the strong peaks appearing at about 21.44 and 23.83 were from the (110) and (200) lattice plane of HDPE crystals, and the new peaks of about $2 \theta=26.44$ were found in GP-0.5/HDPE, corresponding to the $\mathrm{C}(002)$ plane of the graphene. In case of GP-0.5/ CNT-4.0/HDPE sample, the peak intensity of the $\mathrm{C}(002)$ plane became stronger, indicating that carbon nanotubes had similar characteristic peaks to those of the graphenes.

Meanwhile, the characteristic peaks of layered materials became broader or disappeared after the graphene preparation

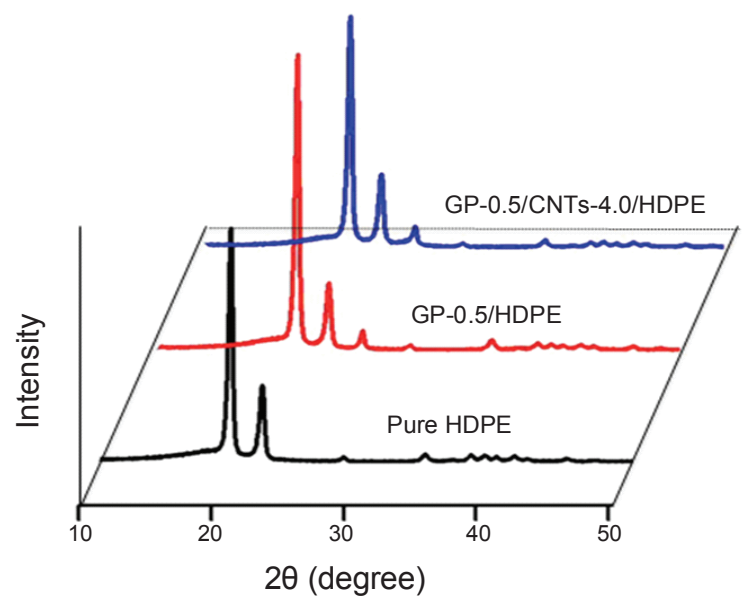

Figure 1. XRD patterns of GP/CNTs/HDPE composites as a function of filler content.

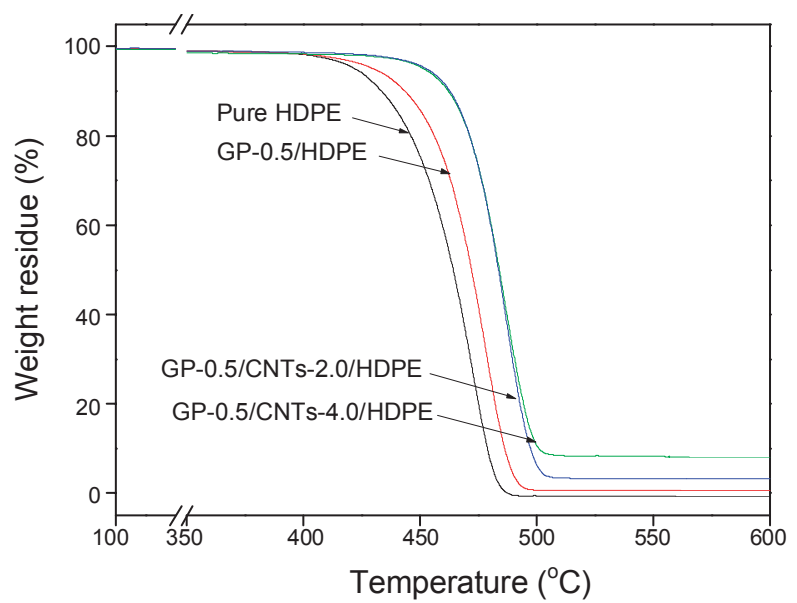

Figure 2. TGA results of GP/CNTs/HDPE composites as a function of filler content.

Table 2. Thermal stabilities of GP/CNTs/HDPE nanocomposites

\begin{tabular}{lcc}
\hline \multicolumn{1}{c}{ Sample name } & $\mathrm{T}_{\max }\left({ }^{\circ} \mathrm{C}\right)$ & IPDT $\left({ }^{\circ} \mathrm{C}\right)$ \\
\hline Pure HDPE & 472.2 & 432.7 \\
GP-0.5/HDPE & 478.1 & 440.9 \\
GP-0.5/CNTs-2.0/HDPE & 486.4 & 487.3 \\
GP-0.5/CNTs-4.0/HDPE & 486.7 & 551.9 \\
\hline
\end{tabular}

due to the severe disorderliness in the polymer matrices. However, in this work, it was found that the peaks of the graphenes were clear and even stronger after adding CNTs. This result indicates that the graphenes have a slightly ordered structure in the matrices and that the adding of CNTs didn't demolish this layered structure, but made it even stronger.

Thermal stabilities. In order to observe the thermal stabilities of the GP/CNTs/HDPE composites in detail, these composites were studied by means of TGA under a nitrogen atmosphere. Figure 2 shows TGA curves of the composites. It was found that pure HDPE didn't have weight residue after $500{ }^{\circ} \mathrm{C}$, indicating 


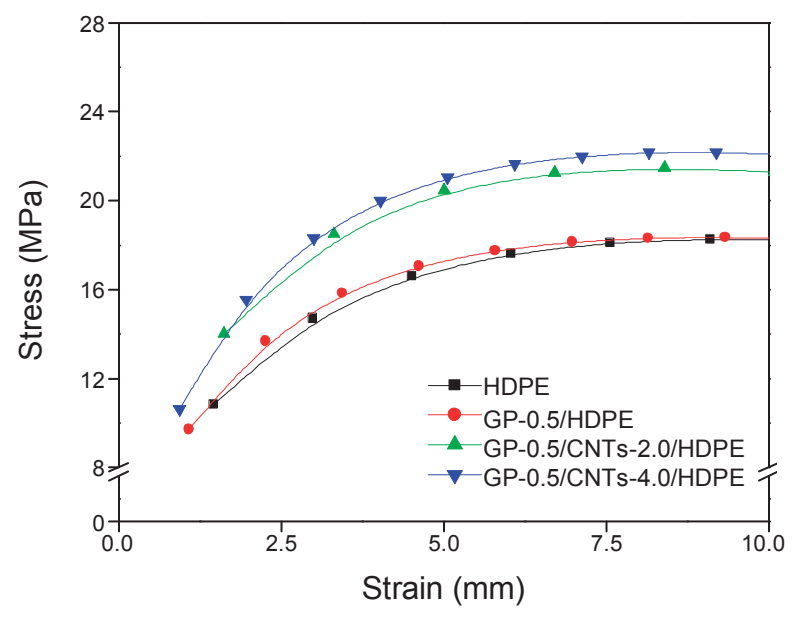

Figure 3. Tensile strength of GP/CNTs/HDPE composites as a function of filler content.

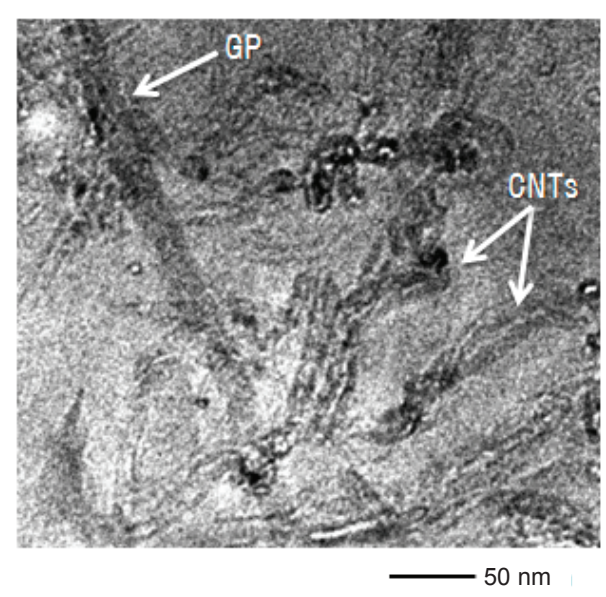

Figure 4. TEM image of GP-0.5/CNTs-4.0/HDPE.

total burn-off. However, carbon-reinforced composites showed quite good thermal stability and weight residue compared to pure HDPE. The thermal stability factors, including the initial procedural decomposing temperature (IPDT) and the temperature at the maximum rate of weight loss $\left(\mathrm{T}_{\max }\right)$, were calculated from the obtained TGA thermograms, and the results were listed in Table 2.

As can be seen, the thermal stability of the composites was significantly enhanced by the addition of GP and CNTs. The $\mathrm{T}_{\max }$ of pure HDPE was at around $472{ }^{\circ} \mathrm{C}$, while those of GP/ HDPE and GP/CNTs/HDPE were $478{ }^{\circ} \mathrm{C}$ and $486{ }^{\circ} \mathrm{C}$, respectively. The IPDT of the composites was $5 \sim 10 \%$ higher than that of the neat HDPE. These results can be interpreted as due to the addition of GP and GP/CNT co-fillers into the HDPE matrix, which increased the surface contact area between the nano carbon fillers and the HDPE matrix, resulting in increased absorption of heat energy during decomposition.

Mechanical properties. The mechanical properties of the nanocomposites were investigated by tensile strength measurements. The tensile strength and elongation of the composites as a function of filler composition are shown in Figure 3. As
Table 3. Specific resistance of GP/CNTs/HDPE nanocomposites

\begin{tabular}{lc}
\hline \multicolumn{1}{c}{ Sample name } & Specific resistivity $(\Omega \cdot \mathrm{cm})$ \\
\hline Pure HDPE & - \\
GP-0.5/HDPE & - \\
CNTs-2.0/HDPE & $6.0 \times 104$ \\
GP-0.5/CNTs-2.0/HDPE & $3.1 \times 102$ \\
GP-0.5/CNTs-4.0/HDPE & $2.9 \times 101$ \\
\hline
\end{tabular}

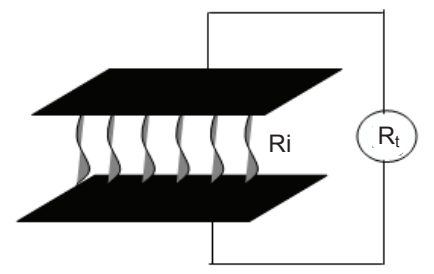

(a)

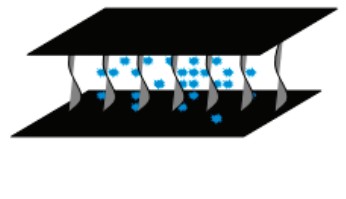

(b)
Figure 5. A scheme of co-filler system in this work; (a) GP-reinforced composites, (b) GP/CNTs co-reinforced composites.

observed, the tensile strength of the composites was increased by the addition of carbon fillers. It is interesting to note that the mechanical strength was significantly enhanced after adding 2.0 wt $\%$ of CNTs in GP-0.5/HDPE, but that strength didn't show further increase in $4.0 \mathrm{wt} \%$ of CNTs. This result indicates that the presence of GP/CNTs co-fillers in the composites leads to a dramatic increase in mechanical properties. These results can be attributed to the fact that the CNTs was finely dispersed between graphene sheets (or layers) in the matrix, thereby increasing the intermolecular interactions, resulting in enhancement of the mechanical properties in the GP/CNTs nanocomposites.

Figure 4 shows a TEM image of GP-0.5/CNTs-4.0/HDPE nanocomposites. It was found that thick graphene layers and CNTs were obviously observed in the HDPE matrix.

Electric properties. The specific resistance of solid materials can be determined by a 4-probe method. Table 3 shows specific resistivity of the composites as a function of filler composition. It was found that the resistivity of pure HDPE and GP-0.5/HDPE samples couldn't be detected, meaning that electric resistivity was higher than $10^{10} \Omega \mathrm{cm}$ (over the limit of the equipment). In the case of the CNTs-2.0/HDPE, the electric resistivity was $6.0 \times 10^{4} \Omega \mathrm{cm}$. Meanwhile, the resistivity of EGs-0.5/CNT2.0/HDPE was dramatically increased and showed $3.1 \times 10^{2}$ $\Omega \mathrm{cm}$. As observed above, the resistivity of GP-0.5/HDPE was not detectable, but the presence of $0.5 \mathrm{wt} \%$ of GP with the same content of CNTs led to predominant enhancement of electric conductivity. This result indicates that mixed GP/CNTs in the matrix formed strong new electric networks that can increase electric conductivity over 100 times more than single CNTs/ HDPE composites. The GP-0.5/CNTs-4.0/HDPE samples also showed good electric conductivity, but this meant an increase of electric network density. Figure 5 illustrates a schematic for the formation of a new electric network by GP and CNTs in this work. As can be seen, the GP in the composites couldn't be connected to each other like broken electric lines. When adding 
CNTs in GP/HDPE composites, each graphene sheet was connected by CNTs, which played the part of bridges to connect each broken point.

\section{Conclusion}

In this work, we proposed a novel co-reinforcement system for conductive polymeric composites. We found that new electric networks were formed and electric conductivity was strongly enhanced when graphenes (GP)/CNTs co-fillers were used. Moreover, the mechanical properties were also increased when co-fillers were used, indicating that GP and CNTs help each other to disperse in the matrix, resulting in enhancement of intermolecular interaction.

Acknowledgments. The authors would like to acknowledge the financial support from GRL Program of Korean Ministry of Education, Science \& Technology.

\section{References}

1. Dhawan, S. K.; Singh, N.; Venkatachalam, S. Synth. Met. 2001, 125,389 .
2. Zhao, X.; Hirigaki, K.; Tabata, I.; Okubayashi, S.; Hori, T. Surf. Coat. Technol. 2006, 201, 628.

3. Schwartz, M. M. Composite Materials Handbook, 2nd ed.; McGraw-Hill: New York, 1992.

4. Donnet, J. B.; Bansal, R. C. Carbon Fibers, 2nd ed.; Marcel Dekker: New York, 1990.

5. Fitzer, E. Carbon Fibers and Their Composites; Springer-Verlag: New York, 1992.

6. Seo, M. K.; Park, S. J. Chem. Phys. Lett. 2004, 395, 44.

7. Kim, B. J.; Park, S. J. J. Colloid Interface Sci. 2007, 315, 791.

8. Kim, H. M.; Kim, K.; Lee, C. Y.; Joo, J. Nanoscale Sci. Design 2004, 84, 589

9. Yan, J.; Fan, Z.; Wei, T.; Qie, Z.; Wang, S.; Zhang, M. Mat. Sci. Eng. B 2008, 151, 174.

10. Zhang, L.; Zhu, H.; Song, Y.; Zhang, Y.; Huang, Y. Mat. Sci. Eng. $B$ 2008, 153, 78 .

11. Mountrichas, G.; Pispas, S.; Tagmatarchis, N. Mat. Sci. Eng. B 2008, 152,40

12. Jou, W. S.; Cheng, H. Z.; Hsu, C. F. J. Alloys Compounds 2007 , $434,641$.

13. Lee, J. H.; Kim, S. K.; Kim, N. H. Scripta Materialia 2006, 55 , 1119.

14. Lei, Y.; Wu, Q.; Clemons, C. M.; Yao, F.; Xu, Y. J. Applied Polym. Sci. 2007, 106, 3958.

15. Lu, W.; Wu, D. J.; Wll, C. L.; Chen, G. H. J. Mater. Sci. 2006, 41, 1785. 